\title{
Scientific Opinion Should be Based on Overwhelming Scientific Literature, and the Door Always Left Open for Change Based on New Findings
}

\author{
B. Gail Demko, DMD, D.ABDSM
}

Sleep Apnea Dentists of New England, Newton Centre, MA

I thank Dr. Hakala for taking the time to read through my long review of the literature and appreciate his concern about some of the topics covered.

\section{SCREENING ALL PATIENTS}

"Dr. Demko discusses the increased mortality and comorbidities associated with sleep-disordered breathing (SDB) but then recommends against dentists screening all patients, even though adding relevant screening questions to a medical history costs essentially nothing. The American Dental Association and other professional groups, including the Minnesota Board of Dentistry, have recommended that dentists screen every dental patient for SDB. Dr. Demko's concern that screening patients for OSA may lead to overtreatment is curious. Hopefully, she does not think this way about screening for other disorders such as periodontal disease or hypertension."

As stated in the article, the ADA policy encourages screening patients for sleep-related breathing disorder (SRBD) as part of a comprehensive medical and dental history to recognize symptoms such as sleepiness, choking, snoring, or witnessed apneas and to evaluate patients for risk factors such as obesity, retrognathia, or hypertension. The Minnesota Board of Dentistry "encourages all dentists screen their patients for obstructive sleep apnea and sleep disorders as part of every routine health history with instruments such as the Epworth Sleepiness Scale and/or other questions...." and agrees clearly with the ADA policy. The ADA goes on to say that those patients should then be referred to the appropriate physicians for proper diagnosis. It must be noted that the position paper includes screening as part of the history a dentist takes on each patient and does not include the use of radiographs or even physical examination. The ADA suggestion of evaluation for the risk factors of weight, retrognathia, and hypertension does require a more "hands-on" approach, factors that I expect all dentists who treat OSA evaluate for routinely ion patients who are at high risk for OSA. Use of questionnaires in a dental practice is all the screening that is required. The article was meant to show the science behind medical screening, the difference between simple questionnaire screening differentiating actions that result in a bill to the patient (such as cone beam computed tomography [CBCT], oximetry, or home sleep apnea testing) from "screening". It was also intended to lower the general dentist expectation that everyone who scores higher than 3 on the STOP-Bang questionnaire will pursue appropriate evaluation, diagnosis, and treatment or that every symptom-free patient in whom OSA is diagnosed requires treatment. As stated in the article, even sleep physicians ascribe only to high-risk screening because there are no data to show that treatment of symptom-free patients with mild OSA will benefit from treatment. For dentists to screen all patients, regardless of symptoms, is akin to measuring blood pressure in all patients regardless of age or medical history. Few 3-yearold children are expected to screen positively for hypertension, but it is possible.

\section{CONE BEAM COMPUTED TOMOGRAPHY}

"Dr. Demko is critical of the use of cone beam computed tomography $(C B C T)$ in the diagnosis and treatment of patients with SDB. Although a cone beam scan alone cannot determine the presence or absence of $S D B$, it can reveal conditions such as nasal septal/turbinate/sinus obstructions, tonsillar and adenoidal hypertrophy, faulty tongue posture, temporomandibular joint misalignment and condylar degeneration, and many other concerns. These conditions may require referral before treatment, can reveal the likelihood of occlusal changes with oral appliance treatment, determine that a patient is simply unsuitable for oral appliance treatment, and provide invaluable assistance in diagnostic and treatment decisions. In addition, CBCT exposes the patient to much less radiation than the conventional panoramic or fullmouth series of radiographs that Dr. Demko 
recommends."

Medical professionals use diagnostic tools such as $\mathrm{CT}$ and magnetic resonance imaging only after a complete clinical evaluation and patient history and professional determination that there is a medical problem. They do not send patients for diagnostic testing as a screening mechanism. Clinical evaluation, including use of a nasal speculum to inspect the internal aspect of the nose, will allow determination of turbinate enlargement or presence of a deviated septum. Many "abnormalities" can be found on a properly evaluated CBCT, but this does not mean there is a disease state present. Many people with a deviated septum have no difficulty breathing and require no treatment; many children outgrow tonsillar enlargement especially if related to an infectious process. Clinical evaluation of both tonsillar enlargement or even adenoid size can be done with direct clinical evaluation or reflection via a dental mirror. Assuming a dentist can justify the use of CBCT--and define it as diagnostic--and the patient complains of chronic nasal congestion and nocturnal nasal congestion, treatment of that issue lies not with the dentist but with a trained otolaryngologist. The trained otolaryngologist will do a complete workup including clinical examination, complete patient history, and evaluation of symptoms. If there is lack of response to medication or lifestyle changes, only then will the physician send the patient for diagnostic radiography. If further treatment is deemed necessary, it is rare that an otolaryngologist will accept a dental CBCT in lieu of a medical CT. If the dentist has billed his "diagnostic" CBCT to medical insurance, then the medically required $\mathrm{CT}$ may not be covered. I caution that many of the conditions for which you are screening with a CBCT are not within the purview of a dentist to treat and are best left to those who are trained to treat such disorders.

The ADA states that, as with other radiographic modalities, "CBCT imaging should be used only after a review of the patient's health and imaging history and the completion of a thorough clinical examination. Dental practitioners should prescribe CBCT imaging only when they expect that the diagnostic yield will benefit patient care, enhance patient safety or improve clinical outcomes significantly". (italics added)

Regarding the level of radiation exposure, the US Food and Drug Administration reports that CBCT does have a radiation dose lower than conventional medical CTs but most CBCT examinations deliver more radiation than conventional dental radiographs. ${ }^{3}$ The Health Physics Society has reported that although CBCT exposes a person to much less radiation than background radiation received cumulatively over a lifetime, the overall radiation exposure depends on the machine used and, measuring in $\mu \mathrm{Sv}$, the dose from a CBCT is 10 to 30 times higher than a digital panoramic equivalent. ${ }^{4}$
Although the use of CBCT is well established in the fields of implant surgery, maxillofacial surgery, and temporomandibular disease, to my knowledge there is no study that has identified any radiographic findings that predict which patients will or will not respond to oral appliance therapy nor identified who will or will not have tooth movement. Other than the dentition being inadequate to support an oral appliance or the patient having complaints of joint disease, there are no published studies that suggest more esoteric and expensive radiologic evaluations are required.

\section{ORTHODONTICS}

"Dr. Demko denies a relationship between orthodontic treatment and the risk of the development of obstructive sleep apnea (OSA). However, a comprehensive conference paper concluded that constricting an upper dental arch clearly increases OSA development, and that "orthodontic expansion of the maxillary dentition is often effective as a fundamental treatment of OSA."

In the study by Maeda et al., ${ }^{5}$ the authors evaluated the plaster casts of 169 adult Japanese men with OSA. The researchers looked at age, body mass index, overjet, overbite, and jaw width ratio (maxillary width/ mandibular width) and found statistically significant correlation only with jaw width ratio (not jaw width alone) and age with the presence of moderate OSA. In a subsequent paper published in $2014^{6}$ these same authors stated that although a narrow maxillary arch was associated with more severe OSA, the maxillary arch width of Japanese males with OSA was not significantly narrower than the Japanese standard value. The authors also noted that the maxillary arch narrowed with an increasing class 2 jaw tendency. Maeda et al. then concluded that although maxillary arch width may aggravate OSA, it is not the maxillary skeletal constriction but rather the maxillary dental arch constriction related to antero-posterior skeletal discrepancy that is related to the presence of OSA. In neither of these studies was orthodontic therapy discussed or considered. Good-quality studies of patients who have undergone extraction orthodontics have not yet shown any relationship to the presence or absence of OSA. ${ }^{7}$ Although the abstract of the paper cited by Dr. Hakala concludes "orthodontic expansion of the maxillary dentition is often effective as a fundamental treatment of OSA" one must note that the entire quote is, "These findings may partially explain why orthodontic expansion of the maxillary dentition is often effective as a fundamental treatment of OSA." To my knowledge and after searching both PubMed and Google Scholar, the only reference to maxillary expansion in adult males was surgical. 


\section{OSA AND GASTROINTESTINAL REFLUX DISEASE}

\begin{abstract}
"Finally, Dr. Demko denies a relationship between OSA and gastrointestinal reflux disease (GERD). Although both are complicated conditions with multiple etiologies and each can occur independently of the other, the literature has established a strong connection between the two. In fact, the prevalence of GERD has been shown to be at $58 \%$ to $62 \%$ in patients with OSA."
\end{abstract}

Dr. Hakala asserts that the Journal of Dental Sleep Medicine article denies a relationship between OSA and GERD; all the article did was to show that the published literature does not support a cause and effect triad of OSA, sleep bruxism, and GERD, only possible comorbidity.

GERD is indeed a very complicated process and it is not within the purview of this letter to go into the intricacies of this problem. I highly recommend the new review article on sleep and nocturnal gastrointestinal reflux (GER) in Chest by Lim et al., now in press. ${ }^{8}$ They review the literature and explain that there is a natural alteration in esophageal physiology during sleep as well as changes in GER mechanisms. Sleep, in general, changes gravitational forces, peristalsis, salivary flow, and muscle tone of the esophageal sphincters, but there is no evidence that the lower esophageal sphincter is pulled open during obstructive events. There is also no linear relationship between breathing events and GER symptom frequency nor between reflux events and arousals from sleep. Lim et al. also state that GER events occur during times of wake (as measured on PSG). Other studies have shown sleep deprivation, regardless of cause, can affect esophageal hypersensitivity and continuous positive airway pressure (CPAP) has been shown to reduce GER even in those without OSA. ${ }^{9}$

Although OSA has a correlation, association, or effect on (even through a mediating process) many pathologic changes (including but not limited to tumor necrosis factor, C-reactive protein, hypertension, stroke, diabetes, atherosclerosis, inflammation, endothelial dysfunction, depression, GER, etc.) the high occurrence of GER in patients with OSA appears related to the common underlying mechanisms, poor muscle tone, altered central nervous system control, distended stomach, or obesity, and an association between two disease states is not the same as cause and effect.

Note: Dr. Hakala cited two supporting pieces of literature. The first was an article by Green et al. that examined questionnaire data from patients with OSA and anamnestic non-GER symptoms (nGER); no measurement of actual GERD was undertaken nor did the physicians refer these patients for evaluation of the GER symptoms. There was improvement in the nGER score in both those who used CPAP (75\%) and in those whose
OSA remained untreated $(31 \%) .{ }^{10} \mathrm{He}$ also cited a letter to the editor by Herr (responding to a previous letter to the editor), which suggested that SDB be evaluated in all children with GERD. ${ }^{11}$ The author of the initial letter to the editor, ${ }^{12}$ a response to a study about chronic cough, asthma, postnasal drip and GERD, ${ }^{13}$ responded that he did not think that was necessary because the causes of OSA in children may not be the same as in adults.

Clinical practice must be based on the whole body of the published literature, verifying that the patient cohort studied is consistent with the conclusions made and data are not taken out of context, with the expectation that there will be changes in conclusions as more data are acquired.

\section{CITATION}

Demko, BG. Scientific opinion should be based on overwhelming scientific literature, and the door always left open for change based on new findings. J Dent Sleep Med. 2019;6(1)

\section{REFERENCES}

1. Resolutions regarding dentists and the diagnosis and treatment of obstructive sleep apnea - Resolution \#1. Minnesota Board of Dentistry. $\quad$ https://mn.gov/boards/assets/Resolution\%20Sleep\%20Apnea_tcm21-78025.pdf. Accessed December 21, 2018.

2. Association Report. The use of cone-beam computed tomography in dentistry. An advisory statement from the American Dental Association Council on Scientific Affairs. J Am Dent Assoc. 2012 Aug;143(8):899-902.

3. Dental Cone-beam Computed Tomography. U.S. Food and Drug Administration. https://www.fda.gov/radiationemittingproducts/radiationemittingproductsandprocedures/medica limaging/medicalx-rays/ucm315011.htm. December 9, 2017 Accessed December 21, 2018

4. Answer to question \#11138 submitted to "Ask the Experts." Health Physics Society https://hps.org/publicinformation/ate/q11138.html. January 12, 2015. Accessed December 21, 2018.

5. Maeda K, Tsuiki S, Inoue Y. (2010). Does dental arch size affect obstructive sleep apnea? https://www.researchgate.net/publication/266771657_Does_Dent al_Arch_Size_Affect_Obstructive_Sleep_Apnea

6. Maaed K, Tsuiki S, Fukada T, Takise Y, Inoue Y. Is maxillary dental arch constriction common in Japanese male adult patients with obstructive sleep apnea? Eur J Orthod. 2014;36(4):403-408.

7. Larsen AJ, Rindal DB, Hatch JP, et al. Evidence supports no relationship between obstructive sleep apnea and premolar extraction: an electronic health records review. $J$ Clin Sleep Med. 2015;11(12):10-15.

8. Lim KG, Morgenthaler TI, Katzka DA. Sleep and nocturnal gastroesophageal reflux and update. Chest. 2018 (in press).

9. Ing $\mathrm{AJ}, \mathrm{Ngu} \mathrm{MC}$, Breslin $\mathrm{AB}$. Obstructive sleep apnea and gastroesophageal reflux. Am J Med. 2000;108(suppl 4a):120S$125 \mathrm{~S}$.

10. Green BT, Broughton WA, O'Connor JB. Marked improvement in nocturnal gastroesophageal reflux in a large cohort of patients with obstructive sleep apnea treated with continuous positive airway pressure. Arch Intern Med. 2003;163(1):41-45.

11. Herr J. Chronic cough, sleep apnea, and gastroesophageal reflux disease 
Chest. 2001;120:1036-1037.

12. Teramoto S. Letter to the Editor. Chest. 2000 Apr;117(4):1215-1216.

13. Palombini BC, Villanova CAC, Araujo E, et al. A pathogenic triad in chronic cough: asthma, postnasal drip syndrome and gastroesophageal reflux disease. Chest. 1999 Aug;116(2): 279-284.

\section{SUBMISSION AND CORRESPONDENCE INFORMATION}

Submitted in final revised form September 27, 2018 Accepted for publication September 27, 2018

Address correspondence to: B. Gail Demko, DMD, 6409 Prairie Dunes Drive, Grand Blanc, MI 48439, Email: bdemko@yahoo.com

\section{DISCLOSURE STATEMENT}

The author has no financial conflicts of interest to disclose. 